\title{
Discharge Against Medical Advice: How Often Do We Intervene?
}

\author{
Jason Edwards, MD*, Ronald Markert, PhD, Dean Bricker, MD
}

Department of Internal Medicine, Wright State University Boonshoft School of Medicine, Dayton, Ohio.

BACKGROUND: Discharges against medical advice (AMA) occur in $1 \%$ to $2 \%$ of hospital stays and are associated with increased morbidity, readmission rates, and 30-day mortality. Risk factors associated with AMA have been investigated, but interventions at the time of discharge have not been carefully examined.

METHODS: We retrospectively reviewed the records of adult patients discharged AMA over a 2-year period from the general medical service of a university-affiliated tertiary care hospital. We assessed for presence of documentation addressing informed consent, patient decision-making capacity, health literacy, follow-up plans, whether medications were prescribed, and whether any warning indicators of impending AMA were apparent.

RESULTS: Two hundred ninety-one records were reviewed. AMA notes were present in 276 (94.8\%) charts. Notes were authored by physicians in 163 (59.1\%) and nurses in 110

Approximately $1 \%$ to $2 \%$ of inpatient stays result in discharges against medical advice (AMA). ${ }^{1}$ Though relatively infrequent, AMA discharges warrant attention as they are associated with higher morbidity, increased risk of readmission, and greater 30-day mortality. $^{2}$ A recent study found a 30 -day readmission rate among AMA patients of $24.5 \%$, nearly twice that of matched non-AMA patients, and a 30-day mortality rate of $1.3 \%$, also nearly double that of planned discharges. ${ }^{3}$ Discharges AMA may be expected to decrease index length of stay, yet accounting for 30day readmissions they are estimated to increase costs $56 \%$ higher than expected from an initial hospitalization. ${ }^{4}$ Patients note several possible reasons for leaving AMA including family emergencies, dissatisfaction with care, financial concerns, or simply feeling better, among others. ${ }^{5-7}$ Risk factors for AMA discharges include previous AMA discharge, having no primary care physician, younger age, lack of insurance, male sex, substance abuse, and lower socioeconomic status. $^{4,6-8}$

\footnotetext{
*Address for correspondence and reprint requests: Jason Edwards, MD, 4881 Sugar Maple Drive, Wright Patterson AFB, OH 45433; Telephone: 765-404-4554; Fax: 937-522-3285; E-mail: jedwards200@ gmail.com

Additional Supporting Information may be found in the online version of this article.

Received: April 15, 2013; Revised: August 18, 2013; Accepted: August 21, 2013

2013 Society of Hospital Medicine DOI 10.1002/jhm.2087

Published online in Wiley Online Library (Wileyonlinelibrary.com).
}

(37.8\%) encounters. Informed consent was present in 88 $(30.2 \%)$ charts, mentioned in the note but not present in the chart in 111 (38.1\%), and not signed in 92 (31.6\%) charts. Decision-making capacity and health literacy were documented in 108 (37.1\%) and 75 (25.8\%) records, respectively. Warning of impending AMA was present in 217 (74.6\%) charts. Medications prescribed and follow-up plans were documented in 71 (24.4\%) and 91 (31.3\%) charts, respectively.

CONCLUSIONS: Advance warning of impending AMA is often present, yet only a minority of cases have prescription of medications or development of follow-up plans. Medical documentation of AMA dispositions is frequently inadequate, suggesting missed opportunities to intervene as well as suboptimal medicolegal scenarios. Journal of Hospital Medicine 2013;8:574-577. (C) 2013 Society of Hospital Medicine

A number of prior studies have assessed risk factors for AMA discharges, the long- and short-term outcomes, patient reasons for leaving, and physician perceptions of why patients leave AMA..$^{3,5,7,9}$ However, there is limited information about opportunities for discharge transition interventions in this potentially more vulnerable population. Because of the increased short-term and long-term risks to these patients, treatment and follow-up plans at the time of discharge may carry even greater importance than follow-up plans with standard discharges. This study analyzed AMA documentation and what interventions were carried out at the time of discharge.

\section{METHODS}

We reviewed the records of all adult patients, ages 18 years and older, admitted to a university-affiliated tertiary care hospital in Dayton, Ohio (a 520-bed hospital with approximately 17,000 adult patient encounters per year) over a 2-year period, and who subsequently left AMA. A hospital database identified 351 adult AMA cases (1.0\% of adult admissions). A single reviewer performed an in-depth review of the 291 patient admissions to the general medical service between January 1, 2009 and December 31, 2010, and manually reviewed and abstracted the data of interest. The Wright State University institutional review board approved the study.

Documentation review focused on the presence of a specified AMA note, the presence of documentation addressing informed consent, patient decision-making capacity, patient health literacy, follow-up plans, 
TABLE 1. Demographic and Hospital Information for 291 Patients Who Left AMA

\begin{tabular}{|c|c|c|}
\hline & $\begin{array}{c}\text { Study Population, } \\
\text { Mean } \pm \text { SD or Count (\%) }\end{array}$ & $\begin{array}{l}\text { Hospital Population, } \\
\text { Mean or Count (\%) }\end{array}$ \\
\hline Age, y & $45.3 \pm 15.9$ & $62.8 \pm 18.2$ \\
\hline \multicolumn{3}{|l|}{ Sex } \\
\hline Male & $168(57.7)$ & $14,965(43.6)$ \\
\hline Female & $123(42.3)$ & $19,333(56.4)$ \\
\hline Length of stay, d & $2.46 \pm 2.82$ & $4.72 \pm 4.74$ \\
\hline 30-day ED re-encounter rate & $121(41.6)$ & \\
\hline 30-day hospital readmission rate & $88(30.2)$ & 4424 (12.9) \\
\hline Prior AMA discharge & $49(16.8)$ & \\
\hline
\end{tabular}

NOTE: Abbreviations: AMA, against medical advice; ED, emergency department; SD, standard deviation.

whether or not medications were prescribed, and whether or not any warning indicators of impending AMA were apparent. These items represented key elements of the discharge policy and procedure in place at our institution during the period of study. We speculated that nurses may be more immediately available at the time of AMA discharge and thus might carry out AMA documentation more often than physicians. To assess this we recorded the role (nurse vs provider) of the writer of AMA notes. We also assessed patient gender, length of stay, prior AMA, 30-day emergency department (ED) re-encounters, and 30-day hospital readmission after AMA discharge.

Informed consent was deemed present if patients signed the hospital's standardized AMA form. Decision-making capacity was assessed as present if there was specific mention of the patient's capacity on the day of discharge. Any mention of health literacy or the patient's stated understanding of his medical condition at any time during the hospitalization was considered positive documentation of healthcare literacy. Follow-up plans included any mention of where and when the patient would return. Discharge medications included prescribed medication or indication that no medications were warranted. Warning indicators included specific mention of the patient's desire to leave AMA. For example, patients who left the unit without informing staff were considered to have given no warning of AMA. Alternatively, when documentation was present stating that the patient had verbally expressed a desire to leave AMA, this was considered advanced warning of AMA.

\section{Statistical Analysis}

Continuous variables were reported as means and standard deviations. Categorical variables were reported as counts and percents. The independent samples $t$ test was used for comparisons involving 2 groups and a second variable measured on a continuous scale. The $\chi^{2}$ test was used to compare 2 categorical variables. Inferences were made at the 0.05 level
TABLE 2. Documentation From Medical Records for 291 AMA Patients

\begin{tabular}{lc}
\hline & Count (\%) \\
\hline AMA note present & $276(94.8)$ \\
Primary AMA note author & \\
Physician & $163(56)$ \\
Nurse, without physician note & $110(37.8)$ \\
Other (ie, social worker) & $3(1.0)$ \\
Warning of impending AMA & $217(74.6)$ \\
Informed consent signed & \\
Yes & $88(30.2)$ \\
No & $92(31.6)$ \\
Absent* & $111(38.1)$ \\
Documentation of decision-making capacity & $108(37.1)$ \\
Documentation of health literacy & $75(25.8)$ \\
Documentation of follow-up plan & $91(31.3)$ \\
Documentation of medications at discharge & $71(24.4)$ \\
\hline
\end{tabular}

NOTE: Abbreviations: AMA, against medical advice.

${ }^{*}$ Absent means mentioned in the notes but not present in the electronic medical record.

of significance with no correction for multiple comparisons.

\section{RESULTS}

Mean age and gender distribution were similar to those reported in other AMA studies (Table 1). ${ }^{3}$ Thirty-day ED revisit and 30-day hospital readmission frequencies for medical service patients were 121 $(41.6 \%)$ and $88(30.2 \%)$, respectively, also similar to those reported in other AMA studies. ${ }^{3}$

Although our intent was to conduct a quantitative assessment of discharge interventions, we found stated reasons for leaving similar to those previously reported. In our study, AMA patients tended to be younger, more likely male, and at increased risk for AMA discharge if they had prior AMA discharges (Table 1). The most common reasons found in the medical record for leaving AMA were caring for sick family members, financial concerns, feeling better, and occasionally dissatisfaction with care, reasons similar to those reported in previous studies. $5,7,9,10$

AMA notes were present in $276(94.8 \%)$ charts. AMA notes were written by physicians in 163 $(59.1 \%)$ and nurses in $110(37.8 \%)$ encounters. The informed consent form was present in 88 (30.2\%) charts, mentioned in the note but not present in the electronic medical record in $111(38.1 \%)$, and not signed in $92(31.6 \%)$ charts. Decision-making capacity and health literacy were documented in 108 $(37.1 \%)$ and $75(25.8 \%)$ records, respectively. Warning of impending AMA was present in 217 (74.6\%) charts. Medications prescribed and follow-up plans were only documented in $71(24.4 \%)$ and 91 (31.3\%) charts, respectively (Table 2 ).

Patients with documentation of medications given did not have decreased 30 -day ED revisits $(33.8 \%$ vs $44.3 \%, P=0.12$ ) or 30 -day hospital readmission $(23.9 \%$ vs $32.4 \%, P=0.18)$. Similarly, there was no 


\begin{tabular}{|c|c|c|c|}
\hline & Yes, \% & No, \% & $P$ Value \\
\hline \multicolumn{4}{|l|}{ Documentation of discharge medications* } \\
\hline 30-day ED revisit & 33.8 & 44.3 & 0.12 \\
\hline 30-day rehospitalization & 23.9 & 32.4 & 0.18 \\
\hline \multicolumn{4}{|l|}{ Documentation of follow-up } \\
\hline 30-day ED revisit & 37.4 & 43.7 & 0.31 \\
\hline 30-day rehospitalization & 29.7 & 30.7 & 0.87 \\
\hline \multicolumn{4}{|l|}{ Physician author of AMA note } \\
\hline 30-day ED revisit & 37.4 & 46.4 & 0.14 \\
\hline 30-day rehospitalization & 28.2 & 31.8 & 0.52 \\
\hline Documentation of medications & 36.2 & 10.0 & $<0.001$ \\
\hline Documentation of follow-up plan & 43.6 & 16.4 & $<0.001$ \\
\hline \multicolumn{4}{|l|}{ Warning of AMA } \\
\hline Documentation of medications & 30.4 & 6.8 & $<0.001$ \\
\hline Documentation of follow-up plan & 37.3 & 13.5 & $<0.001$ \\
\hline
\end{tabular}

NOTE: Abbreviations: AMA, against medical advice; ED, emergency department.

*Medications given at discharge or documentation that medications were not warranted.

relationship between documentation of follow-up plans and 30 -day ED revisits $(37.4 \%$ vs $43.7 \%, P=$ $0.31)$ or 30 -day hospital readmission $(29.7 \%$ vs $30.7 \%, P=0.87)$. Finally, there was no relationship between physician versus nurse authorship of AMA notes and 30-day ED revisits $(37.4 \%$ vs $46.4 \%, P=$ $0.14)$ or 30 -day hospital readmission $(28.2 \%$ vs $31.8 \%, P=0.52$ ) (Table 3).

Physician documentation of the AMA was associated with an increased frequency of discharge medication being prescribed $(36.2 \%$ vs $10.0 \%, P<0.001)$ and with an increased finding of documented followup plans $(43.6 \%$ vs $16.4 \%, P<0.001)$. A documented warning of impending AMA was associated with an increased frequency of discharge medication being prescribed $(30.4 \%$ vs $6.8 \%, P<0.001)$ and increased frequency of follow-up plans being documented $(37.3 \%$ vs $13.5 \%, P<0.001)$ (Table 3$)$.

\section{DISCUSSION}

To gain insights into opportunities for discharge transition interventions in this potentially more vulnerable population, ${ }^{1,5}$ we analyzed AMA documentation and what interventions were carried out at the time of discharge. Our intent was a quantitative assessment of discharge interventions, but we also found stated reasons for leaving AMA that were similar to those previously reported. ${ }^{5,6,10}$

We identified several opportunities for improved documentation as well as targeted discharge intervention among AMA patients. Documentation in the charts of AMA patients was often suboptimal. In our study, a physician's AMA note was present only half of the time. Mention of the patient's mental status or health literacy was present in only one-fourth of cases. Protection from litigation in AMA cases is enhanced when these elements and others, like informed consent, are present in the medical record. ${ }^{11}$
Physician documentation of the AMA was associated with an increased frequency of discharge medication being prescribed and with an increased finding of documented follow-up plans. This association might be confounded by the fact that physicians can prescribe whereas most nurses cannot. The findings that a documented warning of impending AMA was associated with an increased frequency of discharge medication being prescribed $(30.4 \%$ vs $6.8 \%, P<0.001)$ and increased frequency of follow-up plans being documented $(37.3 \%$ vs $13.5 \%, P<0.001)$ suggest opportunities for improvement through early inquiry about potential for AMA as well as early responses when patients threaten to leave AMA.

An important focus of our study was on documentation of discharge medications and follow-up plans. These elements were documented in $31 \%$ and $25 \%$ of charts, respectively. A warning of impending AMA was present in $74.6 \%$ of encounters, yet medications and follow-up plans were documented at a much lower rate. This represents an area where caregivers have the possibility to intervene, but are not documenting that they are doing so. We found no relationship between the documentation of giving prescriptions and giving explicit follow-up plans with decreased rates of return to the $\mathrm{ED}$ or readmission, but that possibility may still warrant future prospective study.

Our study did not attempt to explain why only a minority of AMA discharges include medication prescription or follow-up plans, but a number of potential explanations are possible. Some AMA discharges may occur unannounced with a patient simply walking off the ward giving little or no advance notice. It is also possible that provider perceptions and attitudes toward AMA patients may influence potential interventions. ${ }^{12}$ An AMA discharge is "against" the caregiver's preferred "advice" for the patient, and it may seem illogical to offer patients "second-best" advice. Perhaps some providers have the misconception that medications cannot or must not be prescribed for an AMA discharge. However, second-best therapy may be better than no therapy, and some follow-up better than no follow-up plan.

Given the high rates of ED return and 30-day readmission, the associated increased healthcare costs as well as increased morbidity and mortality associated with AMA dispositions, a continued search for effective intervention strategies and opportunities is warranted. Recently, programs for transition of care/discharge have demonstrated improved outcomes including reduced rates of readmission with standard discharges. ${ }^{13}$ At the time of our study, effective programs such as Project BOOST (Better Outcomes for Older adult through Safe Transitions), ${ }^{14}$ the Care Transitions Program, ${ }^{15}$ and RED (Project Re-engineered Discharge $)^{16}$ were not yet routinely employed, but their common elements may be applicable to the AMA 
population. In general, these programs focus on elements we investigated (patient understanding, followup plans, medications prescribed) but add a number of additional components. Additional elements include written discharge instructions, patient education, teachback process, decision support, emergency plans, caregiver education, telephone follow-up, and transition coaches to coordinate home and office follow-up visits. Most potential interventions add significant time (and cost) to the discharge process. Thus, future studies applying these components to AMA discharges should emphasize timely identification of threatened AMA and prioritized interventions. Future studies should focus on which interventions are the most cost-effective with AMA patients.

Limitations of our study include not being able to access information from area hospitals not in our hospital network, and thus we may not have identified all ED returns and readmissions. Additionally, interventions at the time of discharge (like prescription of medications or provider assessment of decisionmaking capacity) may not have been documented and thus not available for our review. Also, our study was a retrospective review at a single institution and included a relatively small population of patients; consequently, our findings may not apply to other healthcare providers in other hospitals or settings. Our study was strengthened by reviewing all consecutive AMA cases over a 2-year period encompassing a diverse group of healthcare providers.

\section{CONCLUSION}

In the majority of cases reviewed, some advance warning of impending AMA is apparent, affording an opportunity for interventions that may improve health outcomes. Despite this advance warning, only a minority of cases result in key interventions such as prescription of medications or development of followup plans. Medical documentation of AMA dispositions is often inadequate, suggesting missed opportunities for potential intervention as well as suboptimal medicolegal scenarios. Future prospective studies examining cost-effective interventions at the time of AMA discharge and transition of care may provide valuable insight into lowering rates of ED return and rehospitalization.

Disclosures: The views and opinions expressed in this article are those of the author(s) and do not reflect official policy or position of the United States Air Force, Department of Defense, or US government. The authors report no conflicts of interest.

\section{References}

1. Glasgow J, Vaughn-Sarrazin M, Kaboli P. Leaving against medical advice (AMA): risk of 30-day mortality and hospital readmission. J Gen Intern Med. 2010;25(9):926-929.

2. Hwang S, Li J, Gupta R, Chien V, Martin R. What happens to patients who leave hospital against medical advice? CMAJ. 2003;168(4): $417-420$.

3. Southern WH, Nahvi S, Arnsten JH. Increased risk of mortality and readmission among patients discharged against medical advice. Am J Med. 2012;125(6):594-602.

4. Aliyu ZY. Discharge against medical advice: sociodemographic, clinical and financial perspectives. Int J Clin Pract. 2001:56(5):325-327.

5. Onukwugha E, Saunders E, Mullins C, Pradel F, Zuckerman M, Weir M. Reasons for discharges against medical advice: a qualitative study. Qual Saf Health Care. 2010;19:420-424.

6. Jeremiah J, O'Sullivan P, Stein MD. Who leaves against medical advice? J Gen Intern Med. 1995;10:403-405.

7. Green P, Watts D, Poole S, et al. Why patients sign out Against Medical Advice (AMA): factors motivating patients to sign out AMA. Am J Drug Alcohol Abuse. 2004;30:489-493.

8. Ibrahim SA, Kwoh CK, Krishnan E. Factors associated with patients who leave acute-care hospitals against medical advice. Am J Public Health. 2007;97(12):2204-2208.

9. Macrohon B. Pediatrician's perspectives on discharge against medical advice (DAMA) among pediatric patients: a qualitative study. BMC Pediatr. 2012;12:75.

10. Alfandre DJ. "I'm going home": discharges against medical advice. Mayo Clin Proc. 2009:84(3):255-260

11. Levy F, Mareiniss D, Iacovelli C. The importance of a proper againstmedical advice (AMA) discharge: how signing out AMA may create significant liability protection for providers. J Emerg Med. 2012; $43(3): 516-520$.

12. Windish D, Ratanawongsa N. Providers' perceptions of relationships and professional roles when caring for patients who leave the hospital against medical advice. J Gen Intern Med. 2008;23(10): 1698-1707.

13. Enderlin CA, McLeskey N, Rooker JL, et al. Review of current conceptual models and frameworks to guide transition of care in older adults. Geriatr Nurs. 2013;34:47-52.

14. BOOST (Better Outcomes for Older Adults through Safe Transitions). Available at: http://www.hospitalmedicine.org/AM/Template.cfm? Section $=$ Home $\&$ TEMPLATE $=/$ CM $/$ HTMLDisplay.$c f m \&$ CONTENTID $=27659$. Accessed July 29, 2012.

15. The Care Transitions Program. Health care services for improving quality and safety during hand-offs. Eric Coleman model (CTI). Available at: http://www.caretransitions.org. Accessed July 29, 2012.

16. Boston University School of Medicine.Project RED (Re-Engineered Discharge). Available at: http://www.bu.edu/fammed/projectred. Accessed January 13, 2012. 